\title{
INFLUÊNCIA DA BENTONITE NA COMPOSIÇÃO QUÍMICA DE VINHOS QUANDO UTILIZADA PARA ESTABILIZAÇÃO
}

VITOR MANFROI *

FIRMINO SPLENDOR **

LUIZ A. RIZZON ***

JÚLIO MENEGUZZO

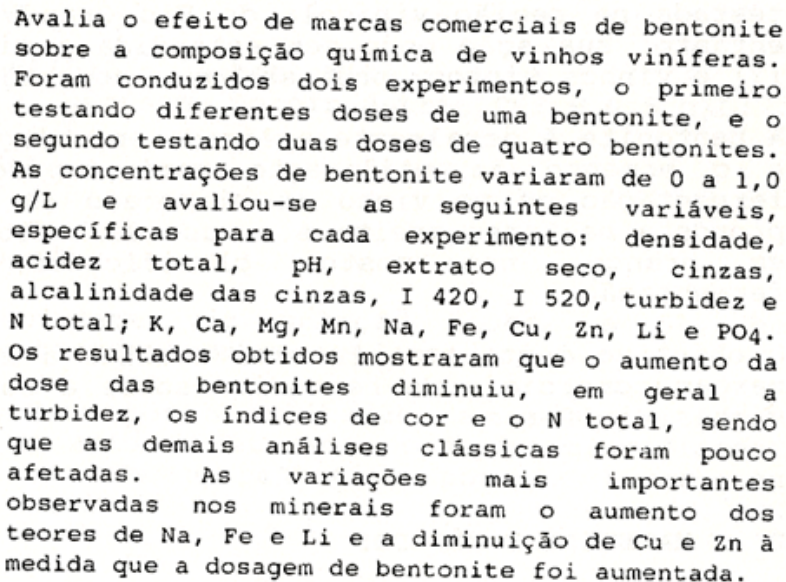

A bentonite é uma substância mineral, obtida da argila do tipo montmorilonita. É constituida principalmente de silicato de aluminio, com quantidades variáveis de magnésio, álcalis, óxido de ferro, etc (6).

\footnotetext{
Eng̊ Agrônomo, M. SC., Escola Agrotécnica Federal Pres. Juscelino Kubitschek, Bento Gonçalves, RS.

** Técnico em Viticultura e Enologia, Escola Agrotécnica Federal Pres. Juscelino Kubitschek, Bento Gonçalves, RS. $\star \star \star$ Eng̊ Agrônomo, Ph. D., Centro Nacional de Pesquisa de Uva e Vinho,
Bento Gonçalves, RS.

**** Engs Agrônomo, Centro Nacional de Pesquisa de Uva e Vinho, Bento Gonçalves, RS.
} 
As propriedades da bentonite se devem a sua capacidade de absorção de água ou outro líquido, aumentando, assim, o volume e formando uma pasta gelatinosa, a qual, devido a sua superfície de contato, apresenta grande capacidade de troca. Possui carga elétrica negativa, que lhe permite flocular os ions metálicos (carregados positivamente) e fixar as particulas coloidais de proteínas, que no $\mathrm{pH} 2,9$ a 3,7 do vinho, são carregadas de eletricidade positiva.

A bentonite é utilizada, principalmente, na clarificação de vinhos brancos, em todas as regiões vitivinícolas do mundo, atuando sobre as enzimas oxidativas, proteínas, microrganismos, matérias tanóides, e prevenindo "casses", principalmente a cúprica. Sua ação desproteinizante, referida como tratamento preferencial pela indústria de enologia, já foi testada na região vinícola do Rio Grande do $\operatorname{sul}(4,5)$. No entanto, sua ação pode ser estendida a vinhos sobreencolados (7) e vinhos tintos, onde se busca aumentar o brilho (2).

A bentonite é geralmente aplicada em solução aquosa de 5-10\%, e o momento de utilização varia, podendo ser antes da fermentação ou no vinho já elaborado (3). Recentemente, uma proposta bastante aceita é o uso da bentonite na vinificação em branco, nos mostos clarificados, antes mesmo da fermentação.

o objetivo deste trabalho foi avaliar a influência de quatro marcas comerciais de bentonite sobre a composição química de vinhos viniferas.

- trabalho foi realizado na cantina da Agroindústria CVE, da Escola Agrotécnica Federal Presidente Juscelino Kubitschek, e no Centro Nacional de Pesquisa de Uva e vinho (CNPUV/EMBRAPA), em Bento Gonçalves, RS.

Foram realizados dois experimentos, utilizando quatro marcas comerciais de bentonite (numeradas de 1 a 4), disponíveis no setor enológico da Micro-Região Geográfica de Caxias do Sul.

\subsection{DOSES DE BENTONITE SOBRE A COMPOSIÇÃO DE VINHOS BRANCO E} TINTO VINÍFERAS

Este experimento foi conduzido no delineamento completamente casualizado, com sete tratamentos, e três repetições. os tratamentos foram aplicados em vinhos branco e tinto viníferas, já estabilizados pelo frio, cujas características são apresentadas na Tabela 1 . 


\begin{tabular}{lcr}
\hline & Vinho branco & Vinho tinto \\
\hline - turbidez & 8,68 & 312,00 \\
- álcool ( $\left.{ }^{\circ} \mathrm{GL}\right)$ & 11,20 & 10,50 \\
- acidez total (meq/L) & 78,00 & 85,00 \\
\hline
\end{tabular}

Os tratamentos foram:

1) testemunha (sem aplicação de bentonite);

2) bentonite padrão 2, usada na Agroindústria CVE - 0,5 g/L;

3) bentonite $1-0,2 \mathrm{~g} / \mathrm{L}$;

4) bentonite $1-0,4 \mathrm{~g} / \mathrm{L}$;

5) bentonite $1-0,6 \mathrm{~g} / \mathrm{L}$;

6) bentonite $1-0,8 \mathrm{~g} / \mathrm{L}$ e

7) bentonite $1-1,0 \mathrm{~g} / \mathrm{L}$.

A bentonite foi aplicada via solução aquosa a $5 \%$ em recipientes de vinho branco, com capacidade de 1 L.

As variáveis analisadas após a aplicação da bentonite foram realizadas uma semana após a retirada do vinho clarificado, que ficou em contato com a bentonite por 72 horas. As variáveis estudadas foram:

1) turbidez;

2) I 420 (vinho branco);

3) I 520 (vinho tinto) e

4) minerais: $\mathrm{Mg}, \mathrm{Na}, \mathrm{Fe} \mathrm{e} \mathrm{Li}$.

\subsection{EFEITO DE DIFERENTES BENTONITES SOBRE A COMPOSIÇÃO DE VINHO BRANCO VINÍFERA}

Após o primeiro ensaio pensou-se em aumentar o espectro de bentonites, restringindo, no entanto, as doses utilizadas. Neste experimento trabalhou-se com quatro bentonites, utilizando-se duas concentrações: $0,5 \mathrm{~g} / \mathrm{L}$ e $1,0 \mathrm{~g} / \mathrm{L}$, comparando-se ainda com uma testemunha, onde não foi aplicada bentonite.

Aqui foram motivo de análise as seguintes variáveis:

1) análises clássicas: densidade, acidez total, pH, extrato seco, cinzas, alcalinidade das cinzas, I 420 e $\mathrm{N}$ total;

2) componentes minerais: $\mathrm{K}, \mathrm{Ca}, \mathrm{Mg}, \mathrm{Mn}, \mathrm{Fe}, \mathrm{Cu}, \mathrm{Zn}, \mathrm{Li}, \mathrm{PO}_{4}$. 
3.1 EFEITO DE DOSES DE BENTONITE SOBRE A COMPOSIÇÃO DE VINHOS BRANCO E TINTO VINÍFERAS

Os valores referentes às variáveis analisadas constam nas Tabelas 2 e 3 .

TABELA 2 - TURBIDEZ E ÍNDICES DE COR AVALIADOS EM VINHOS BRANCO E TINTO APÓS TRATAMENTO COM DIFERENTES DOSES DE BENTONITE

\begin{tabular}{|c|c|c|c|c|c|c|c|c|}
\hline \multirow[t]{2}{*}{ Doses de Bentonite } & \multicolumn{4}{|c|}{ Vinho Branco } & \multicolumn{4}{|c|}{ Vinho Tinto } \\
\hline & Turbidez. & * & $1+20$ & & Turbidez & & I 520 & \\
\hline & $* *$ & & & & & & & \\
\hline Testemunha & 8.82 & a & $0 .+30$ & a & 321.00 & a & 0.517 & a \\
\hline Bentonite $2-0.5 \mathrm{~g} / \mathrm{L}$ & 2.03 & $\mathrm{~b}$ & 0.0 .57 & $\mathrm{~b}$ & 56.80 & b & 0.388 & bc \\
\hline Bentonite $1-(1.2 \mathrm{~g} / \mathrm{L}$ & 1.74 & b & 0.058 & b & 53.00 & b & $0 . .397$ & $\mathrm{~b}$ \\
\hline Bentonitc $1-(1.4 \mathrm{~g} / \mathrm{L}$ & 1.01 & b & 0.052 & b & 48.80 & b & $0.37 \%$ & $\mathrm{bc}$ \\
\hline Bentonitc $1-0.6 \mathrm{~g} / \mathrm{L}$ & 0.97 & b & 0.045 & b & 3.60 & b & 0.345 & $b c$ \\
\hline Bentonitc $1-(1.8 \mathrm{~g} / \mathrm{L}$ & 0.86 & b & $0.04+4$ & b & 2.10 & b & 0.338 & $\mathrm{c}$ \\
\hline Bentonite $1-1.0 \mathrm{~g} / \mathrm{L}$ & 1.70 & b & 0.065 & b & 1.50 & b & 0.334 & c \\
\hline
\end{tabular}

* Valores transformados segundo raiz $(x+1)$.

** Mćdias seguidas por Ictras distintas diferem entre si pelo teste de Tukẹ ao nível de $5 \%$ de probabilidade.

A turbidez indicou, no vinho branco, que as doses de bentonite utilizadas permitiram clarificá-lo de forma satisfatória. Percebeu-se que a dose mais baixa da bentonite 1 apresentou algo de turbidez, o mesmo ocorrendo com a dose de $1,0 \mathrm{~g} / \mathrm{L}$, cujo vinho permaneceu com particulas de clarificante em suspensão. No vinho tinto a turbidez passou de valores elevados na testemunha, para valores cada vez mais baixos de acordo com o aumento da dose utilizada; as doses mais baixas e a bentonite padrão apresentaram turbidez ainda um pouco elevadas. Estes dados são semelhantes aos encontrados por outros autores que trabalharam com mostos (1).

Nos índices de cor, o vinho branco apresentou diferença significativa no I 420 em todas as doses de bentonite, em relação a testemunha; o valor da testemunha pode ter sido influenciado pela turbidez do vinho. No vinho tinto houve, basicamente, três grupos, quanto ao I 520, representados pela testemunha, as doses intermediárias $(0,2 ; 0,4 ; 0,6 \mathrm{~g} / \mathrm{L}$ da bentonite 1 , e da padrão), e as doses mais elevadas da bentonite $1(0,8$ e $1,0 \mathrm{~g} / \mathrm{L})$. Os valores obtidos para I 520 mostraram decréscimo de cor à medida que se aumentou a dose de bentonite, confirmando a suspeita de que a bentonite "retira" a cor dos vinhos tintos. 
TABELA 3 - MINERAIS AVALIADOS EM VINHOS BRANCOS E TINTOS APÓS TRATAMENTO COM DIFERENTES DOSES DE BENTONITE

\begin{tabular}{|c|c|c|c|c|c|c|c|c|}
\hline \multirow[t]{2}{*}{ Doses de Bentonite } & \multirow{2}{*}{\multicolumn{2}{|c|}{$\frac{\mathrm{Mg}}{\mathrm{mg} / \mathrm{L}}$}} & \multirow{2}{*}{\multicolumn{2}{|c|}{$\frac{\mathrm{Na}}{\mathrm{mg} / \mathrm{L}}$}} & \multirow{2}{*}{\multicolumn{2}{|c|}{$\frac{\mathrm{Fc}}{\mathrm{mg} / \mathrm{L}}$}} & \multicolumn{2}{|l|}{$\mathrm{Li}$} \\
\hline & & & & & & & $\mu \mathrm{g} / \mathrm{L}$ & $\therefore$ \\
\hline \multicolumn{9}{|c|}{ Vinho Branco } \\
\hline & $*$ & & & & & & & \\
\hline Tesıcmunha & 53 & a--- & 39.5 & $\cdots d$ & 3.6 & $\cdots \mathrm{d}$ & 5.7 & $\ldots \uparrow$ \\
\hline Bentonitc $2-0.5 \mathrm{~g} / \mathrm{L}$ & 53 & 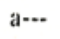 & 48.6 & $a b--$ & 4.3 & ab-- & 7.3 & --cd-- \\
\hline Bentonitc $1-0.2 \mathrm{~g} / \mathrm{L}$ & 53 & $a \cdots$ & 41.4 & $--\mathrm{cd}$ & 3.8 & -cd & 6.4 & $\cdots \mathrm{cf}$ \\
\hline Bcintonitc $1-0.4 \mathrm{~g} / \mathrm{L}$ & 52 & a-.. & +5.2 & -bc- & 4.0 & -bcd & 7.0 & ---dc- \\
\hline Bentonitc $1-0.6 \mathrm{~g} / \mathrm{L}$ & 53 & a-.. & +6.0 & -bc- & 4.1 & $-b c-$ & 8.0 & -bc--- \\
\hline Bentonite $1-0.8 \mathrm{~g} / \mathrm{L}$ & 53 & a-.. & +9.6 & ab-- & 4.2 & ab-- & 8.3 & -b-... \\
\hline Bentonitc $1-1.0 \mathrm{~g} / \mathrm{L}$ & 53 & $\mathrm{a} \cdots$ & 51.1 & a-.. & +.6 & a-.. & 9.1 & a...... \\
\hline \multicolumn{9}{|c|}{ Vinho Tinto } \\
\hline Testcmunha & 92 & $a-\cdots$ & 37.3 & $\cdots d$ & 3.7 & $\cdots d$ & 3.5 & $\cdots+n$ \\
\hline Bcntonitc $2-0.5 \mathrm{~g} / \mathrm{L}$ & 92 & a $\cdots$ & 48.4 & -b-- & 3.8 & $--c d$ & 4.8 & - cd- \\
\hline Bentonitc $1-0.2 \mathrm{~g} / \mathrm{L}$ & 93 & 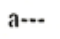 & 39,3 & $\cdots \mathrm{cd}$ & 3.8 & $--c d$ & 4.0 & $\cdots-\cdots$ \\
\hline Bentonitc $1-0.4 \mathrm{~g} / \mathrm{L}$ & 93 & a... & +1.6 & -bc- & t.0 & $-b c-$ & 4.7 & $\cdots d-$ \\
\hline Bentonitc $1-0.6 \mathrm{~g} / \mathrm{L}$ & 93 & a-.- & +4.5 & $-b c-$ & 4.0 & $-b c-$ & 5.1 & $--c--\cdot$ \\
\hline Bentonitc $1-0.8 \mathrm{~g} / \mathrm{L}$ & 92 & a-.- & 48.4 & $-b \cdot-$ & +.1 & ab-- & 5.9 & -b--..- \\
\hline Bentonite $1-1.0 \mathrm{~g} / \mathrm{L}$ & 93 & $\mathrm{a} \cdots$ & 54.2 & a-.. & +.1 & a-.. & 6.3 & a-.... \\
\hline
\end{tabular}

* Médias seguidas por letras distintas diferem centre si pelo teste de Tukey ao nível de 5\% de probabilidade.

Quanto aos minerais, o teor de Mg não foi afetado por nenhuma dose de bentonite, nos dois tipos de vinho, comprovando o pouco acréscimo deste cátion quando se utiliza este clarificante.

Os teores de $\mathrm{Na}$ elevaram significativamente à medida que se aumentou a dose. Em ambos os vinhos, o $\mathrm{Na}$ foi o cátion mais afetado pela incorporação do clarificante. Isto é explicado pela própria constituição da bentonite, em cuja composição entra este elemento, sendo que este aumento está plenamente justificado quanto aos valores anteriormente encontrados (4, 8 ).

O Fe, a exemplo do $\mathrm{Na}$, teve seus teores elevados à medida que se aumentou as doses, havendo diferenças significativas entre as mesmas. As diferenças foram mais acentuadas no vinho branco, o que prova a maior suscetibilidade dos vinhos brancos à incorporação deste cátion.

O Li também foi afetado pela incorporação das bentonites, e o aumento dos teores acompanhou o aumento das doses do clarificante. Como para $\mathrm{Fe}$, o Li também foi afetado em maior grau no vinho branco, ainda que o teor inicial deste vinho fosse mais elevado na testemunha. 
Neste experimento nāo foi realizada a análise estatística, e os dados são apresentados, de maneira separada, para cada uma das concentrações de bentonite utilizadas, nas Tabelas 4, 5, 6 e 7 .

TABELA 4 - INFLUÊNCIA NA COMPOSIÇÃO DAS ANÁLISES CLÁSSICAS DE UM VINHO BRANCO ESTABILIZADO COM $0,5 \mathrm{~g} / \mathrm{L}$ DE QUATRO MARCAS COMERCIAIS DE BENTONITE

\begin{tabular}{|c|c|c|c|c|c|}
\hline \multirow[t]{2}{*}{ Análise clássica } & \multirow[b]{2}{*}{ Testcmunha } & \multicolumn{4}{|c|}{ Bentonite } \\
\hline & & 1 & 2 & 3 & 4 \\
\hline & * & & & & \\
\hline Densidade a $20^{\circ} \mathrm{C}$ & 0.9937 & 0.9940 & $0.99+4)$ & 0.9936 & 0.9939 \\
\hline Acidez total (meq/L) & 120.0 & 118.6 & 118.0 & 118.0 & 118.0 \\
\hline $\mathrm{pH}$ & 2.98 & 2.98 & 2.98 & 2.97 & 2.97 \\
\hline Extrato scco $(\mathrm{g} / \mathrm{L})$ & 21,46 & 21.46 & 21.46 & 21.33 & 21.86 \\
\hline Cinzas $(\mathrm{g} / \mathrm{L}$ & 1.68 & 1.61 & 1.58 & 1.73 & 1.63 \\
\hline Alcalinidade das cinzas $(m c q / L)$ & 13,0 & 12.9 & 12.5 & 13.5 & 12.5 \\
\hline $\mathrm{N}$ total $(\mathrm{mg} / \mathrm{L})$ & 118.0 & 105.9 & 104.5 & $10+.6$ & 100.8 \\
\hline $1+20$ & 0.098 & 0.092 & 0.090 & 0.088 & 0.090 \\
\hline
\end{tabular}

* Os valores representam a média de três repctições

TABELA 5 - INFLUÊNCIA NA COMPOSIÇÃO DAS ANÁLISES CLÁSSICAS DE UM VINHO BRANCO ESTABILIZADO COM 1,0 g/L DE QUATRO MARCAS COMERCIAIS DE BENTONITE

\begin{tabular}{|c|c|c|c|c|c|}
\hline \multirow[t]{2}{*}{ Análise clássica } & \multirow[b]{2}{*}{ Testemunla } & \multicolumn{4}{|c|}{ Bentonitc } \\
\hline & & 1 & 2 & 3 & 4 \\
\hline & * & & & & \\
\hline Densidade a $20^{\circ} \mathrm{C}$ & 0.9937 & 0.9938 & 0.9940 & 0.9939 & 0.9937 \\
\hline Acidez total $(\mathrm{mcq} / \mathrm{L})$ & 120,0 & 118.6 & 118.0 & 117.3 & 118.0 \\
\hline $\mathrm{pH}$ & 2,98 & 2.99 & 2.98 & 2.98 & 2.97 \\
\hline Extrato scco $(\mathrm{g} / \mathrm{L})$ & 21.46 & 21.33 & 21.06 & 21.20 & 20.93 \\
\hline Cinzas $(\mathrm{g} / \mathrm{L}$ & 1.68 & 1.66 & 1.83 & 1,61 & 1.61 \\
\hline Alcalinidade das cinzas (meq/L) & 13.0 & 13.2 & 13.5 & 13.4 & 12.0 \\
\hline $\mathrm{N}$ total $(\mathrm{mg} / \mathrm{L})$ & 118.0 & 100,8 & 93.8 & 102.2 & - \\
\hline$I+20$ & 0.098 & $0 .(189)$ & 0.089 & 0.085 & 0.187 \\
\hline
\end{tabular}

* Os valores representam a média de três repetiçòes 
Com relação ao pH, extrato seco, cinzas e alcalinidade das cinzas, as variações provocadas nos vinhos pela utilização de bentonite foram inexpressivas, e pequenos aumentos foram observados nos valores da densidade.

A redução observada no teor de acidez total é decorrência do grau de alcalinidade da bentonite, uma vez que as suspensốes preparadas na água apresentam $\mathrm{pH}$ compreendido entre 8,0 e 9,0 .

Com relação ao $\mathrm{N}$ total, a aplicação de doses normais de bentonite nos vinhos para a sua estabilização $(0,3$ a $1,0 \mathrm{~g} / \mathrm{L})$ é, segundo alguns autores, responsável pela diminuição de 8 a $15 \%$ nos teores deste elemento. No experimento realizado, o teor médio de $\mathrm{N}$ total passou de $118,00 \mathrm{mg} / \mathrm{L}$ para 103,95 e $98,93 \mathrm{mg} / \mathrm{L}$ quando as concentrações de bentonite foram de 0,5 e $1,0 \mathrm{~g} / \mathrm{L}$, respectivamente.

- tratamento com bentonite foi responsável, também pela diminuição do índice de cor ( $(420$ ), o que já havia ocorrido no experimento anterior, e já referido em outras citaçōes (2, $8)$.

TABELA 6 - INFLUÊNCIA NA COMPOSIÇÃO MINERAL DE UM VINHO BRANCO ESTABILIZADO COM $0,5 \mathrm{~g} / \mathrm{L}$ DE QUATRO MARCAS COMERCIAIS DE BENTONITE

\begin{tabular}{|c|c|c|c|c|c|}
\hline \multirow{2}{*}{$\begin{array}{l}\text { Mincral } \\
(\mathrm{mg} / \mathrm{L})\end{array}$} & \multirow[b]{2}{*}{ Testcmunha } & \multicolumn{4}{|c|}{ Bentonite } \\
\hline & & 1 & 2 & 3 & 4 \\
\hline & $*$ & & & & \\
\hline $\mathrm{K}$ & 59t & 59) 1 & 60.3 & 593 & 507 \\
\hline $\mathrm{Na}$ & 9.6 & 19.2 & 25.3 & 18.3 & 19.6 \\
\hline $\mathrm{Ca}$ & 75.2 & 76.7 & 79,8 & 83.9 & 76.2 \\
\hline $\mathrm{Mg}$ & 59.1 & 60.5 & 62.4 & 60.2 & 60.8 \\
\hline $\mathrm{Mn}$ & 1.1 & 1.0 & 1.2 & 1.3 & 1.3 \\
\hline $\mathrm{Fc}$ & 1,2 & 1.9 & 1.6 & 1.6 & 1.7 \\
\hline $\mathrm{Cu}$ & 3.6 & 1,0 & 1.1 & 1.1 & 0.9 \\
\hline $\mathrm{Zn}$ & 1.5 & 1,0 & 1,0 & 1.0 & 1.0 \\
\hline $\mathrm{Li}^{* *}$ & 5.1 & 10.0 & 6.7 & 10.1 & $11 . .3$ \\
\hline $\mathrm{PO}_{4}$ & 182.7 & 186.0 & 185.7 & 183.6 & 183,3 \\
\hline
\end{tabular}

* Os valores represcntam a média de três repetições.

** Os valores do litio correspondenı a $\mu \mathrm{g} / \mathrm{L}$. 

$\begin{aligned} \text { TABELA } 7 \text { - INFLUENCIA NA COMPOSIÇÃO MINERAL DE UM VINHO } & \text { BRANCO ESTABILIZADO COM } 1,0 \mathrm{~g} / \mathrm{L} \text { DE QUATRO MARCAS } \\ & \text { COMERCIAIS DE BENTONITE }\end{aligned}$

\begin{tabular}{|c|c|c|c|c|c|}
\hline \multirow{2}{*}{$\begin{array}{l}\text { Mineral } \\
(m \mathrm{~g} / \mathrm{L})\end{array}$} & \multirow[b]{2}{*}{ Testcmunlas } & \multicolumn{4}{|c|}{ Bentonitc } \\
\hline & & 1 & 2 & 3 & 4 \\
\hline & $*$ & & & & \\
\hline K & 594 & 597 & 608 & 616 & 500 \\
\hline $\mathrm{Na}$ & 9.6 & 15.7 & 19.8 & 14.4 & 15.7 \\
\hline $\mathrm{Ca}$ & 75.2 & 77.8 & 79.2 & 79.2 & 76.6 \\
\hline $\mathrm{Mg}$ & 59.1 & 61.1 & 62.4 & 60.0 & $6(1) .9$ \\
\hline Mn & 1.1 & 1.1 & 1.2 & 1.2 & 1.3 \\
\hline $\mathrm{Fc}$ & 1.2 & 1.7 & 1.6 & 1.4 & 1.6 \\
\hline $\mathrm{Cu}$ & 3.6 & 3.5 & 2.1 & 1.6 & 1.7 \\
\hline$Z_{n}$ & 1.5 & 2.0 & 1.3 & 1.2 & 1.2 \\
\hline $\mathrm{Li} * *$ & 5.1 & 8.7 & 6.3 & 8.2 & 9.11 \\
\hline $\mathrm{PO}_{4}$ & 182.7 & 179.9 & $18+.5$ & 185.4 & 183.9 \\
\hline
\end{tabular}

* Os valores representam a média de três repetições.

** Os valores do lítio correspondem a $\mu \mathrm{g} / \mathrm{L}$.

Quanto aos componentes minerais, o tratamento do vinho com bentonite provocou aumento da quantidade de $\mathrm{Na}$, aumento este que foi mais importante naquelas amostras que receberam dose maior de bentonite. A variação no teor de $\mathrm{Na}$ observada, a exemplo do primeiro experimento, confirmam que as quatro marcas de bentonite testadas sāo do tipo sódico.

outro cátion que apresentou aumento importante nos vinhos tratados com bentonite foi o Li. O aumento médio observado foi de aproximadamente 58,8 e $86,3 \%$, quando as doses aplicadas foram de 0,5 e $1,0 \mathrm{~g} / \mathrm{L}$, respectivamente.

Já ○ $\mathrm{K}$, ○ $\mathrm{Ca}$, ○ $\mathrm{Mg}$, ○ $\mathrm{Mn}$ e $\circ \mathrm{P}$ também aumentaram com a utilização de bentonite. Neste caso, no entanto, as variações foram de menor importância.

Relativamente ao $\mathrm{Fe}$, a bentonite, de modo geral, não tem ação para precipitar este elemento no vinho, uma vez que é encontrado, principalmente no estado de íons complexos carregados negativamente. O aumento de $\mathrm{Fe}$ observado nos vinhos tratados é consequiente da liberação deste elemento pelas bentonites utilizadas.

Com relação ao Cu e $\mathrm{Zn}$, constatou-se diminuição em relação aos teores iniciais destes elementos encontrados na testemunha; a quantidade precipitada aumentou com a dose de bentonite utilizada. A diminuição, principalmente de $\mathrm{Cu}$, reduz os efeitos deste elemento sobré possíveis alterações dó vinho, em especial a "casse" cúprica, fato já demonstrado em outros relatos $(2,8)$. 
- Nos vinhos brancos, as doses crescentes de bentonite diminuiram a turbidez e o I 420, apesar de que no primeiro ensaio, a dose mais elevada apresentou problemas de suspensão de partículas do clarificante;

- No vinho tinto, as doses crescentes de bentonite também diminuíram a turbidez e o I 520;

- As demais análises clássicas foram pouco afetadas, à excessão do teor de $\mathrm{N}$ total que diminuiu seus valores com o uso de bentonite;

- Quanto a compostos minerais, as variações mais importantes foram observadas com $\mathrm{Na}$, $\mathrm{Fe} e \mathrm{Li}$. os teores destes elementos aumentaram com o aumento da dose de bentonite empregada;

- A bentonite se mostrou eficiente para reduzir os teores de $\mathrm{Cu}$, reduzindo, em boa parte, a possibilidade de "casse" cúprica.

\begin{abstract}
It analyses the effect of commercial marks of bentonite in the chemical composition of vinifera wines. Two experiments were carried out. The first test used differents concentrations of one bentonite, and second test used two concentrations of four bentonites. The concentration of bentonite were from 0 to $1.0 \mathrm{~g} / \mathrm{L}$, and it was evaluated the following variables, especific to each experiment: density, total acidity, pH, dry extract, ashes, alcalinity of ashes, I 420, I 520, turbidity and total $\mathrm{N}$; $\mathrm{K}, \mathrm{Ca}, \mathrm{Mg}, \mathrm{Mn}, \mathrm{Na}, \mathrm{Fe}, \mathrm{Cu}, \mathrm{Zn}, \mathrm{Li}$ e $\mathrm{PO}_{4}$. The results showed that the increasing of the bentonite concentration reduced, in general, the turbidity, the color index and the total N. The other classic analyses were a little bit affected. The most important variation observed in the minerals were the concentration increasing of $\mathrm{Na}, \mathrm{Fe}$ and $\mathrm{Li}$ and the reduction of $\mathrm{Cu}$ and $\mathrm{zn}$.
\end{abstract}

\title{
REFERÊNCIAS BIBLIOGRÁFICAS
}

1 CASTINo, M., UBIGLI, M. Esperienze sull'impiego della bentonite nello sfecciamento dei mosti. L'Enotécnico. n. giugno, p. 673-81, 1986.

2 CAPELLERI, G. La clarificacion frente al problema de la estabilizacion, limpidez y claridad. La Semana Vitivinicola, n. 1901 , p. $166-73,1983$. 
3 EWART, A.J.W., PHIPPS, A.J., ILLAND, P.G. Bentonite additions to wine: before, during or stater fermentation? The Australian Grapegrover \& Winemaker, v. 17, n. 196, p. 46-47-54, 1980 .

4 FAGGION, V. Estudo das bentonites e suas açōes nos vinhos brancos. Bento Gonçalves : Centro Nacional de Uva e Vinho / Embrapa, 1993. 29 p. Relatório de estágio datilografado.

5 FENOCCHIO, P., PESSI, G.N. Poder desproteinizante de uma bentonita. Pelotas : EMBRAPA, 1975. 8 p. (Boletim Técnico, 97)

6 PARONETTO, L., DAL CIN, A.J. Prodotti Chimici nella Tecnica Enologica. Verona : Scuola D'Arte Tipográfica D. Bosco, 1954. 458 p.

7 PEYNAUD, E., RIBEREAU-GAYON, J., RIBEREAU-GAYON, P. La clarificacion de los vinos. La Semana Vitivinicola, n. 2044 , p. 3823-31, 1985.

8 RIBEREAU-GAYON, J., PEYNAUD, E., RIBEREAU-GAYON P. SUDRAUD, P. Sciences et techniques du vin. Paris : Dunod, 1977. V. 4 .

Agradecimentos

Os autores agradecem as Técnicas em Enologia Magda Beatris Gatto Salvador e Nilda Maria Gatto zucco, pela colaboração prestada na realização das análises. 